\title{
biomolecules
}

ISSN 2218-273X

www.mdpi.com/journal/biomolecules/

Review

\section{RNase P-Mediated Sequence-Specific Cleavage of RNA by Engineered External Guide Sequences}

\section{Merel Derksen ${ }^{1,2, \dagger}$, Vicky Mertens ${ }^{1, \dagger}$ and Ger J.M. Pruijn ${ }^{1,2, *}$}

1 Department of Biomolecular Chemistry, Institute for Molecules and Materials, Radboud University, P.O. Box 9101, Nijmegen NL-6500 HB, The Netherlands; E-Mails: M.Derksen@ncmls.ru.nl (M.D.); vicky.mertens@student.ru.nl (V.M.)

2 Department of Biomolecular Chemistry, Radboud Institute for Molecular Life Sciences, Radboud University, P.O. Box 9101, Nijmegen NL-6500 HB, The Netherlands

$\dagger$ These authors contributed equally to this work.

* Author to whom correspondence should be addressed; E-Mail: G.Pruijn@ncmls.ru.nl; Tel.: +31-243-616-847.

Academic Editor: Denis Drainas

Received: 29 July 2015 / Accepted: 29 October 2015 / Published: 9 November 2015

\begin{abstract}
The RNA cleavage activity of RNase P can be employed to decrease the levels of specific RNAs and to study their function or even to eradicate pathogens. Two different technologies have been developed to use RNase P as a tool for RNA knockdown. In one of these, an external guide sequence, which mimics a tRNA precursor, a well-known natural RNase P substrate, is used to target an RNA molecule for cleavage by endogenous RNase P. Alternatively, a guide sequence can be attached to M1 RNA, the (catalytic) RNase P RNA subunit of Escherichia coli. The guide sequence is specific for an RNA target, which is subsequently cleaved by the bacterial M1 RNA moiety. These approaches are applicable in both bacteria and eukaryotes. In this review, we will discuss the two technologies in which RNase P is used to reduce RNA expression levels.
\end{abstract}

Keywords: external guide sequence; RNase P; RNA cleavage; RNA knockdown; RNA targeting 


\section{Introduction}

For many years, strategies to interfere with gene expression post-transcriptionally have been applied to study gene function in cultured cells. Moreover, the knockdown of specific RNAs is an attractive approach for the treatment of microbial infections. Currently, multiple agents and techniques (e.g., antisense oligonucleotides and oligonucleotide analogues, ribozymes, DNAzymes and RNA interference) are available to reduce the levels of specific RNAs. In general, all these approaches involve the targeting of an RNA by an antisense nucleic acid, followed by recognition and subsequent cleavage of the duplex by an endogenous or exogenous enzyme.

RNase $\mathrm{P}$ is an example of a ribozyme that can be employed for the knockdown of specific RNAs. This enzyme was first discovered in Escherichia coli (E. coli) over forty years ago and has since been identified in almost all bacteria, archaea and eukarya. The universal function of RNase P is the removal of the 5'-leader sequence from tRNA precursors (pre-tRNAs; Figure 1A) [1], but a wide range of other substrates, especially in prokaryotes, has been identified since then (reviewed in [2]).

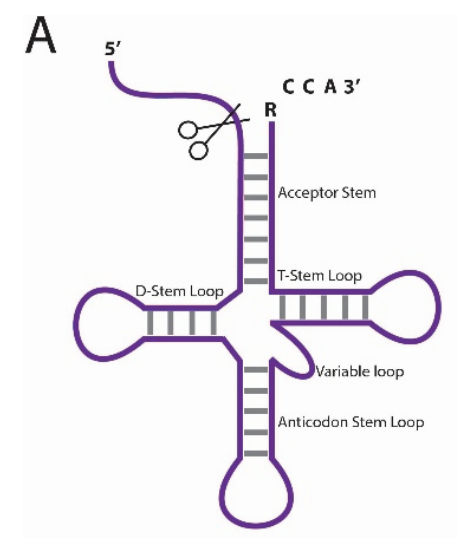

D

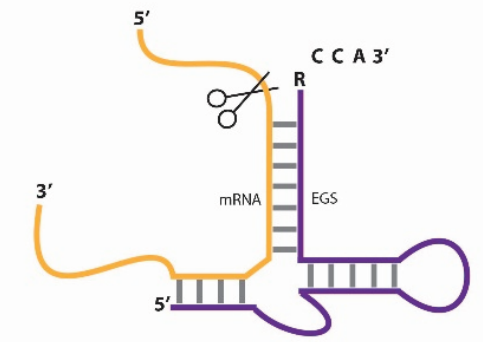

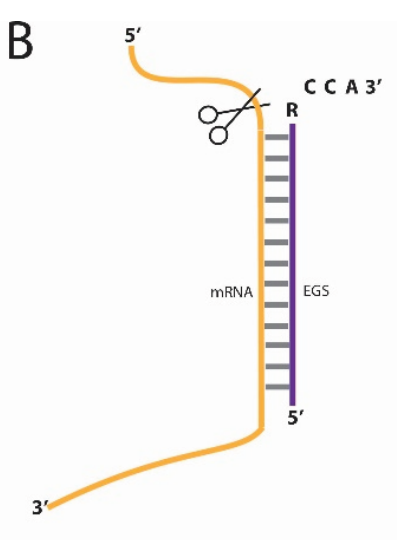
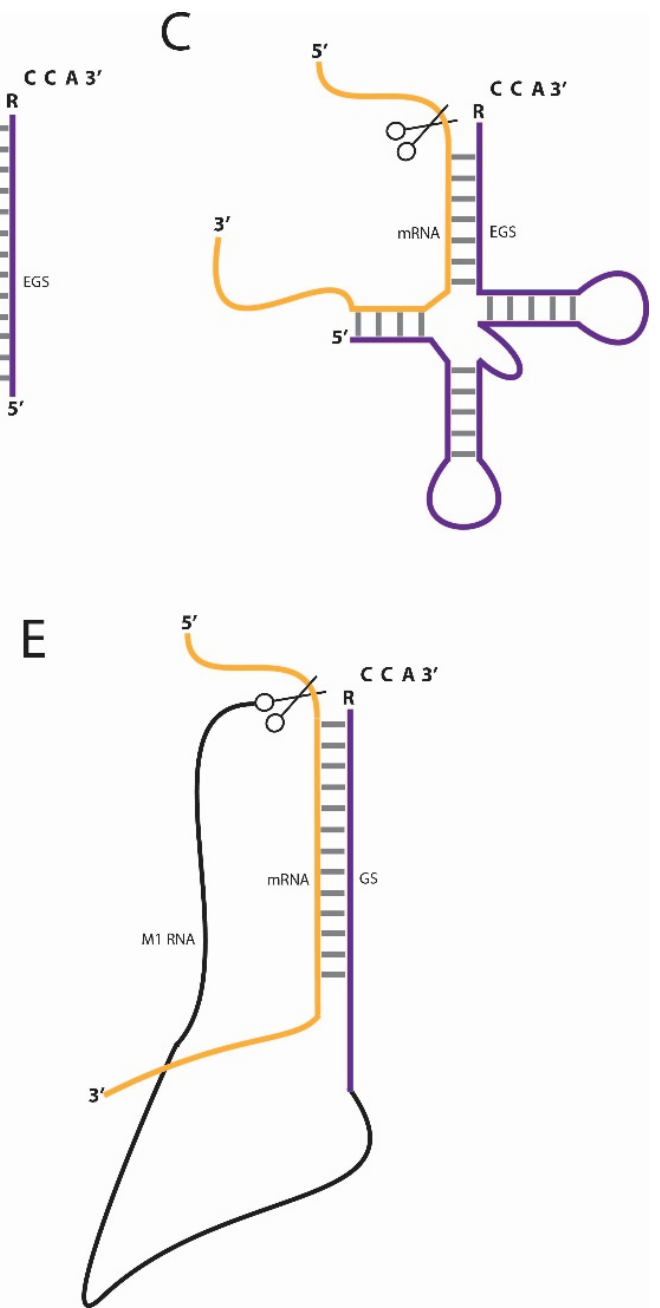

Figure 1. Schematic illustration of RNase P-based substrate cleavage. (A) Pre-tRNA, a natural substrate of RNase P; (B) The bacterial stem-only EGS-mRNA substrate; (C) The EGS-mRNA complex resembling $3 / 4$ of pre-tRNA for eukaryotic target cleavage; (D) The minimized eukaryotic EGS-mRNA complex and (E) an M1GS-mRNA complex. The RNase P cleavage activity is indicated with the scissors. 
In most organisms, RNase $\mathrm{P}$ is a ribonucleoprotein (RNP) complex composed of a single RNA molecule and one or more protein subunits. The RNA subunit of the RNP complexes in bacteria, archaea and eukaryotes displays catalytic activity in vitro in the absence of the protein subunits, which has been demonstrated for the RNA subunit from E. coli, Bacillus subtilis, methanobacteria, extreme halophiles, thermococci, Giardia lamblia, and humans [3-5]. More recently, protein-only forms of RNase P (PRORP, proteinaceous RNase P) have also been found and these act in the nucleus and/or organelles of plants, some protists, algae, as well as in animal mitochondria [6-10]. Interestingly, replacing the Saccharomyces cerevisiae RNase P RNA component by PRORP only led to minor changes in tRNA processing and, furthermore, no increase in putative RNase P substrates was observed [11].

The ability of RNase P to cleave pre-tRNA and other RNA substrates can be used to degrade specific RNA molecules. Nearly 30 years ago, Altman and coworkers found that a model substrate, which resembles the acceptor stem of a pre-tRNA, was cleaved by E. coli RNase P in vitro [12]. Soon, it was found that a bimolecular substrate composed of two complementary RNAs was also efficiently cleaved in vitro by the bacterial RNase P [13]. The discovery that artificial substrates can be cleaved by RNase P led to the development of different techniques in which either endogenous or exogenous RNase $\mathrm{P}$ is employed to knockdown specific RNAs. These techniques generally involve (external) guide sequences, which target specific RNAs and resemble the structure of the pre-tRNA substrates after base-pairing.

In this review, we will discuss the current techniques for RNA down-regulation that are based on RNase P activity. The application of these techniques will be illustrated by recent examples aimed at the down-regulation of microbial genes, genes involved in cancer and a (human) host gene that is involved in HIV1 infection.

\section{Approaches in RNase P-Mediated RNA Knockdown}

The use of RNase P in RNA downregulation is widely applicable and is often used in bacterial as well as eukaryotic cells [14,15], and to a lesser extent in plant cells [16,17]. Early applications in E. coli were based on an external guide sequence (EGS) that forms a stem structure with the target RNA (Figure 1B) [14,18], the endogenous RNase P recognizes this stem structure and cleaves the target RNA at the junction from single-stranded to double-stranded regions. The stem is usually between 13 and 16 base pairs long and is fused to the essential RCCA sequence at the 3'-end of the EGS, which is also present on natural pre-tRNA substrates in E. coli. The two cytidines in the 3 '-RCCA sequence are bound by a GG sequence pair in the catalytic domain of RNase P RNA. This base pairing interaction was found to be essential for the catalytic activity in E. coli [19].

Unlike bacterial RNase P, the eukaryotic RNase P enzyme cannot cleave the relatively simple stem structure. Initial studies showed that in eukaryotes the EGS-target RNA duplex has to mimic 3/4 of a pre-tRNA structure, which lacks only the D-loop (Figure 1C). Subsequent in vitro selection studies showed that the EGS can be further minimized (Figure 1D), which results in an EGS-target RNA duplex mimicking a pre-tRNA lacking the anticodon-stem and -loop structures; furthermore, substitutions of nucleotides in the T-loop render the EGS inactive [20,21]. Since many eukaryotes produce pre-tRNAs lacking the RCCA sequence at the 3'-end, this element may not be essential for RNase P activity in 
eukaryotes. Indeed, it was shown both in vitro and in human cell cultures that the presence of the RCCA sequence on the EGS is not necessary for RNA cleavage [15,22].

While EGS technologies use the endogenous enzyme for target cleavage, in the M1 guide sequence (M1GS) approach the guide sequence is linked to the E. coli RNase P RNA, termed M1 (Figure 1E) [23,24]. The guide sequence forms a stem structure with the target RNA, similar to the EGS-substrate complex, and is cleaved by the conjugated M1 RNA. In E. coli, M1 RNA requires the $\mathrm{C} 5$ protein for RNase $\mathrm{P}$ activity, but, surprisingly, this does not seem to be the case in eukaryotes lacking the C5 protein. A major disadvantage of the M1GS compared to the EGS approach is the length of the RNA to be transfected. Compared to the M1GS RNAs, EGS RNAs are relatively short and can be easily synthesized and therefore modified to increase stability and delivery.

\section{Stabilization and Delivery of EGS/M1GS RNAs}

Due to their highly specific guide sequences, the EGS and M1GS technologies provide attractive approaches for the treatment of microbial infections, cancer and genetic diseases. However, as with other gene interference therapies, the delivery of RNAs is complicated because of multiple reasons. First, the RNA molecules may elicit innate immune responses; second, RNAs are nuclease sensitive and thus easily degraded in vivo; third, due to the negative charge of the RNA, the RNA molecules do not pass easily through cell membranes; and fourth, the RNAs generally need to be targeted to a specific tissue or cell type. Various efforts have been made to overcome some of the problems for in vivo delivery of the EGSs and M1GSs.

\subsection{Improved Nuclease Resistance}

EGS molecules can be chemically modified to make them less nuclease-sensitive [22,25-27]. The nucleotides in the EGS can be replaced, for example, by 2'-O-methyl derivatives to decrease RNase recognition and cleavage of the EGS molecules. Residues in the minimized EGS molecule, except in the loop corresponding to the T-loop of tRNA, can be substituted without loss of RNase P activity in both in vitro and in vivo studies [22,25]. In addition, phosphodiamidate morpholino oligonucleotides (PMO), which are not sensitive to cellular nucleases due to their unnatural backbone, can be used [26]. Furthermore, the addition of a 3'-3' inverted thymidine at the 3'-end of the EGS provides protection to 3'-exonucleases [25].

Besides substitution of nucleotides with 2'-O-methyl derivatives, oligomers composed of LNA/DNA residues can be used to improve nuclease resistance [27]. An EGS completely made up of DNA residues did not elicit RNase P cleavage in vitro, in contrast to co-oligomers of LNA and DNA residues. Substitution of DNA with LNA increased the thermal stability of the EGS-mRNA duplex, but the number of substitutions did not correlate with the cleavage activity in vitro. Interestingly, the introduction of LNA residues at the 3'-CCA and several other specific positions throughout the EGS led to high cleavage activity. These co-oligomers were also resistant to bacterial RNase treatment. Substitution of the 3'-CCA probably increases the stability of the interaction with M1 RNA, whereas the other LNA residues may improve the interaction with the target mRNA. The authors also demonstrated that these co-oligomers can reduce target mRNA levels in a permeable strain of E. coli at a concentration of 
$50 \mathrm{nM}$. Unfortunately, the DNA/LNA oligomer was not taken up by a wild type strain of $E$. coli and therefore methods to improve internalization of these molecules need to be developed.

\subsection{EGS and MIGS Delivery}

Two methods, using either conjugates of a cell-penetrating peptide (CPP) with a PMO or attenuated Salmonella vectors, have been developed to improve the delivery of EGS and M1GS molecules. Due to the relatively small size of EGSs, they can be easily synthesized as PMO with a modified 3 '-end that can be linked to a positively charged CPP, to improve cellular uptake [28]. Altman and coworkers have shown that both the CCP and the CPP-PMO EGS directed against the bacterial gyrA gene, which encodes a DNA gyrase, leads to a decrease in bacterial survival. Moreover, the CPP and CPP-PMO EGS have a combined effect on bacterial death [29], although the additional effect of the CPP-PMO EGS did not appear to be very pronounced. In another study, the effects of conjugating EGSs to another CPP on target cleavage were studied. The CPP-PMO EGSs appeared to cleave target RNAs at non-predicted sites. The reasons for this miscleavage are not understood, but the positively charged peptide may interact with either the target RNA or the M1 RNA and alter the overall structure of the EGS-RNase P complex [28]. CPP-PMO EGSs have been used to kill both bacteria and malaria parasites and might become useful antibiotics [28-31].

M1GSs are approximately 400 nucleotides in length and therefore chemical synthesis will result in low yields. As a consequence, it is hard to introduce the stabilizing chemical modifications as discussed above. For the delivery of M1GSs attenuated Salmonella bacteria can be used. A plasmid containing a sequence encoding the M1GS is introduced into an attenuated Salmonella strain, which contains mutations that reduce the virulence and facilitate intracellular lysis of the bacteria and release of the transgene construct. Subsequently, this leads to efficient expression of the delivered gene in the target cells. Advantages of this system are that Salmonella can be targeted to specific cell types, for example macrophages, and that non-invasive oral administration is possible. This system has already been demonstrated to be successful in cell culture and mice for the delivery of both M1GSs and EGSs [32-34], although the mechanism by which the nucleic acid is transferred to the host remains unclear. Although not applied yet, virus-based gene delivery systems are obviously suitable as well for the introduction of M1GS molecules in target cells.

\section{Examples of RNase P-Based RNA Knockdown}

In this paragraph, we will illustrate the use of EGS and M1GS methods by describing a number of studies in which they were successfully applied in various cells and organisms to repress the levels of target RNAs. The general goal of most of these studies was to interfere with microbial proliferation and the targets can be roughly divided into four categories, bacterial, parasitic, viral and host transcripts that are involved in microbial replication. M1GSs have also been used to specifically target and inhibit growth of cancer cells. Examples of each of these categories will be discussed and are summarized in Table 1. 
Table 1. Overview of literature data on the application of the external guide sequence (EGS) and M1GS technologies towards infectious agents and cancer.

\begin{tabular}{|c|c|c|c|c|c|}
\hline Organism/Infectious Agent & Target Molecule & EGS/M1GS & Effect & System Tested & Reference \\
\hline \multirow{6}{*}{ E. coli } & \multirow{3}{*}{ bla, cat } & EGS & Decreased viability & Cultured E. coli & [35] \\
\hline & & CPP-PMO EGS & Decreased viability & Cultured E. coli & {$[28,30]$} \\
\hline & & LNA/DNA EGS & Decreased viability & Cultured E. coli & [27] \\
\hline & gyrA, rпрA & EGS & Decreased viability & Cultured E. coli & [36] \\
\hline & gyrA & CPP-PMO EGS & Decreased viability & $\begin{array}{l}\text { Cultured E. coli and } \\
\text { various other bacteria }\end{array}$ & {$[28-30]$} \\
\hline & fts $Z$ & CPP-PMO EGS & Induced filimentation and $\sim 10$-fold decreased viability ${ }^{1}$ & Cultured E. coli & [37] \\
\hline S. aureus & gyrA & CPP-PMO EGS & $\begin{array}{l}\text { Accelerated epithelialization and wound } \\
\text { closure and smaller eschar formation }\end{array}$ & $\begin{array}{l}\text { Cultured } \text { S. aureus; infected murine } \\
\text { cutaneous wound model }\end{array}$ & [38] \\
\hline S. enterica & $\operatorname{inv} B /$ inv $C$ & EGS & Reduced host-invasion & Salmonella invasion in Henle-407 cells & [39] \\
\hline F. tularensis & $m g l B$ & EGS & Reduced mRNA levels & $m g l B$ expression in $E$. coli culture & [40] \\
\hline Y. pestis & $y s c N, Y s c S$ & EGS & Reduced mRNA levels & $\begin{array}{c}y s c N \text { and } y s c S \text { expression } \\
\text { in } E \text {. coli culture }\end{array}$ & [41] \\
\hline P. falciparum & PfGyrA & CPP-PMO EGS & $63 \%-75 \%$ growth inhibition measured in different strains & P. falciparum infected erythrocytes & [31] \\
\hline Influenza virus & $N P, P B 2$ & EGS & $\begin{array}{c}70 \%-95 \% \text { reduced expression of the viral M1 protein } \\
\text { with the use of two EGSs simultaneously }\end{array}$ & Mouse $\mathrm{C} 127$ cells & [42] \\
\hline \multirow{3}{*}{$\begin{array}{l}\text { Herpes simplex } \\
\text { virus } 1\end{array}$} & ICP4 & M1GS & 1000 -fold reduced virus titer $(36 \mathrm{~h}, \mathrm{MOI} 2)$ & $\psi \mathrm{CRE}$ cells (NIH 3T3 cells) & [43] \\
\hline & & Minimized EGS & $80 \%$ decrease in mRNA and $75 \%$ decrease in protein level & Human 143tk- cells & [44] \\
\hline & $T K$ & $\begin{array}{l}\text { Minimized EGS variant, } \\
\text { improved by in vitro selection }\end{array}$ & $96 \%$ decrease in mRNA and $95 \%$ decrease in protein level & Human 143 tk- cells & [45] \\
\hline
\end{tabular}


Table 1. Cont.

\begin{tabular}{|c|c|c|c|c|c|}
\hline Organism/Infectious Agent & Target Molecule & EGS/M1GS & Effect & System Tested & Reference \\
\hline \multirow{10}{*}{$\begin{array}{l}\text { Human } \\
\text { cytomegalo-virus }\end{array}$} & \multirow{4}{*}{ IE1, IE2 } & WT M1GS & 500 -fold reduced virus titer (5 days, MOI 1 ) & Human U373MG cells & [46] \\
\hline & & $\begin{array}{l}\text { M1GS-variant G224A, } \\
\text { G225A }\end{array}$ & 3000 -fold reduced virus titer ( 5 days, MOI 1 ) & Human U373MG cells & [47] \\
\hline & & M1GS—variant U80C, C188U & 10,000-fold reduced virus titer ( 5 days, MOI 1 & Human U373MG cells & [48] \\
\hline & & M1GS_variant A94G, G194C & 3500 -fold reduced virus titer (5 days, MOI 1 ) & Human U373MG cells & [49] \\
\hline & \multirow{6}{*}{$\mathrm{AP} / \mathrm{PR}$} & Minimized EGS & 500 -fold reduced virus titer (4 days, MOI 1 ) & Human foreskin fibroblasts & [50] \\
\hline & & Minimized EGS & 800 -fold reduced virus titer (5 days ,MOI 2 ) & Human U373MG cells & [51] \\
\hline & & Minimized EGS variant C321 & 7000 -fold reduced virus titer ( 5 days, MOI 2 ) & Human U373MG cells & [52] \\
\hline & & M1GS & 100 -fold reduced virus titer (5 days, MOI 3 ) & Human U373MG cells & [53] \\
\hline & & M1GS & 2000-fold reduced virus titer (5 days) & Human U373MG cells & [54] \\
\hline & & M1GS—variant A81C, G194A & 50,000 -fold reduced virus titer ( 5 days, MOI 1 ) & Human U251 cells & [55] \\
\hline \multirow{2}{*}{$\begin{array}{l}\text { Murine } \\
\text { cytomegalo-virus }\end{array}$} & \multirow{2}{*}{$\mathrm{AP} / \mathrm{PR}$} & M1GS; Salmonella SL101 & $\begin{array}{l}\text { 2500-fold reduced virus titer ( } 4 \text { days, MOI 1) } \\
\text { in macrophages; prolonged survival of mice }\end{array}$ & Mouse J774 macrophages; mice & [33] \\
\hline & & $\begin{array}{l}\text { Minimized EGS; } \\
\text { Salmonella SL201 }\end{array}$ & $\begin{array}{l}\text { 3000-fold reduced virus titer (4 days) in macrophages; } \\
\text { prolonged survival of mice }\end{array}$ & Mouse J774 macrophages; mice & [34] \\
\hline \multirow{2}{*}{ Hepatitis B virus } & $\begin{array}{l}\text { pgRNA, pre-S/L mRNA, } \\
\text { S mRNA }\end{array}$ & $\begin{array}{c}\text { Minimized EGS variant C418; } \\
\text { Salmonella SL301 }\end{array}$ & $\begin{array}{l}\text { 2000-fold reduced viral DNA level in HepG2.2.15 cells ( } 3 \text { days) } \\
\text { and 200,000-fold reduced viral DNA level in mice ( } 5 \text { days) }\end{array}$ & HepG2.2.15 cells; mice & [56] \\
\hline & $\begin{array}{l}\text { pgRNA, pre-S/L mRNA, } \\
\text { S mRNA }\end{array}$ & $\begin{array}{c}\text { Minimized EGS variant C386; } \\
\text { Salmonella SL201 }\end{array}$ & 6000-fold reduced viral DNA level (4 days) & HepG2.2.15 cells & [57] \\
\hline Hepatitis $\mathrm{C}$ virus & HCV 5'-UTR & M1GS & $>1000$-fold reduced virus titer (1 day, MOI 1) & Human Huh7.5.1 cells & [58] \\
\hline \multirow{2}{*}{$\begin{array}{c}\text { Human } \\
\text { immuno-deficiency virus } 1\end{array}$} & LTR and TAT & Minimized EGS & Reduced $\mathrm{p} 24$ protein levels & COS cells & [59] \\
\hline & TAT region & M1GS — variant G83U, G340A & 150-fold reduced virus titer (12 days) & Human H9 cells & [60] \\
\hline \multirow[b]{2}{*}{ Humans } & CCR5 & Minimized EGS & 50-fold reduced level of HIV p24 (12 days) & Human PM1 cells & [61] \\
\hline & BCR-ABL & M1GS & $\begin{array}{l}96 \% \text { and } 97 \% \text { cell death in respectively, } \\
\text { p190 and p210 dependent cells }\end{array}$ & $\begin{array}{l}\mathrm{Ba} / \mathrm{F} 3 \text { cells expressing } \\
\text { the } \mathrm{p} 190 \text { and } \mathrm{p} 210 \text { oncogenes }\end{array}$ & [62] \\
\hline
\end{tabular}




\subsection{Downregulation of Bacterial RNAs and Infections}

Early studies on the use of EGSs for downregulation of bacterial RNAs showed that EGSs can lead to targeted cleavage of, amongst others, the LacZ mRNA both in vitro and in vivo [14,18]. More recent research focused on the downregulation of antibiotic resistance gene transcripts by EGSs. Downregulation of these transcripts can convert antibiotic-resistant strains into antibiotic-sensitive strains and might therefore become useful tools in combatting infections with resistant bacteria. Alternatively, EGSs have been used that target bacterial genes involved in essential processes, like gyrA, and in virulence, like Salmonella invB and invC.

\subsubsection{Drug Resistance Genes}

Altman and coworkers were the first to show that EGSs can be used to increase the sensitivity to antibiotics in ampicillin- and chloramphenicol-resistant E. coli [35]. An EGS expression system was developed in which the EGS, fused at the 3'-end to a hammerhead sequence, was expressed from a plasmid containing a T7 promoter and terminator. After transcription by T7 RNA polymerase, the hammerhead-mediated cleavage ensured the correct formation of the 3'-end of the EGS. Using this system, it was demonstrated that higher levels of the EGS as well as the expression of multiple distinct EGSs correlate with drug sensitivity. However, for obvious reasons, the delivery of a plasmid carrying the EGS into bacteria in an infected mammal will be difficult. Therefore, CPP-PMO EGSs targeting ampicillin and chloramphenicol resistance genes have been developed and these have been shown to decrease viability of ampicillin- and chloramphenicol-resistant E. coli $[28,30]$. Interestingly, reduced viability was observed both in the presence and in the absence of the antibiotics. It is likely that the reduced viability in the absence of antibiotics is due to off-target effects of the CPP-PMO EGS. Indeed, the CPP-PMO EGS targeting the chloramphenicol resistance gene appeared to be fully complementary to several E. coli genes. This observation implies that the capacity of the technique to eliminate bacteria extends beyond the matches with specific gene sequences. However, since the CPP-PMO EGSs seem to target multiple unintended RNAs, a major drawback of this method might be the concomitant targeting of host genes and the resulting effects on the host.

Another class of drug resistance genes are the AAC(6')-I type acetyltransferases which inactivate the antibiotic amikacin. These genes are widely disseminated and the AAC(6')-Ib enzyme especially limits the therapeutic use of amikacin [63]. Multiple EGSs were designed based on RNase H mapping of single-stranded RNA regions in the AAC(6')-Ib mRNA [64]. Subsequent in vitro experiments led to a selection of five EGSs based on binding affinity to the mRNA and induction of cleavage by RNase P. In vivo studies were performed in which the EGSs were expressed from a plasmid using the T7/hammerhead approach mentioned above [35]. EGSs with the highest binding affinities in vitro most efficiently decreased amikacin-resistance in vivo. The partial repression of amakicin-resistance, however, indicated that EGSs may aid, but may not completely eliminate infections, and therefore it has been suggested that they might be particularly useful in combination with other drugs [64]. Later, the EGS that reduced the amikacin-resistance most efficiently, was further developed to become a nuclease resistant LNA/DNA oligomer [27]. 


\subsubsection{Essential Genes}

Besides drug resistance genes, two essential genes, gyrA and $r n p A$, have been extensively investigated as bacterial EGS targets. The gyrA gene encodes gyrase A, a type II topoisomerase, and $r n p A$ encodes the RNase P C5 protein. Nearly 15 years ago, Mc Kinney and coworkers designed two EGSs targeting gyrA and two EGSs targeting rnpA. Expression of a single EGS targeting gyrA reduced E. coli growth approximately 7-fold, whereas the combination of both EGSs targeting gyrA resulted in a 10-fold reduction. The combination of all gyrA and rnpA EGSs reduced E. coli growth 26-fold [36]. These results confirmed the observations by Altman and coworkers that the effects of multiple EGSs on bacterial survival are additive [35]. McKinney et al. also showed that 1 to 3 mismatches in the EGS-mRNA duplex did not affect its inhibitory capacity, whereas five mismatches abolished this effect completely [36].

To achieve delivery into the bacteria, various CPP-PMO EGSs targeting gyrA have been used [28-30]. A CPP-PMO EGS targeting a conserved region of gyrA acts as a general antibiotic at micromolar concentrations for both Gram-positive and Gram-negative bacteria [29]. Since three mismatches are allowed in the EGS-mRNA duplex, it is possible to use a single gyrA EGS on multiple bacteria. In addition, the natural microbiome of the host might be affected. Nevertheless, it was shown that a CPP-PMO EGS targeting the Staphylococcus aureus gyrA mRNA led to improved wound healing of a S. aureus infected wound in mice [38]. Altogether, due to their effective delivery, CPP-PMO EGSs targeting essential genes are promising tools for the elimination of various bacterial infections, such as surface wound infections or internal infections, for which they can be administered orally or intravenously. Moreover, CPP-PMO EGSs may become especially useful in treating infections with antibiotic resistant bacteria.

Targeting of another bacterial gene, fts $Z$, was investigated by Sala and coworkers [37]. fts $Z$ is the most highly conserved bacterial cell division gene coding for the proto-ring protein FtsZ, which recruits other proteins to the divisome and generates the constrictive force to initiate cell division. Because the $f t s Z$ gene has no homology to eukaryotic genes, it is an attractive target for EGS-mediated downregulation. Target regions in the E. coli fts $Z$ gene were based upon the results of bioinformatic prediction of the mRNA secondary structure. Two regions were selected as good candidates, because they were predicted not to be associated with stable secondary structures. Three EGSs were designed and they all induced efficient RNase P-mediated cleavage in vitro. To test the activity in vivo, one of the EGSs was expressed using the T7/hammerhead system. This EGS showed activity in E. coli, because filamentation and growth impairment were observed. Nuclease-resistant EGSs that can interfere with FtsZ expression still need to be prepared. In addition, the direct delivery of these EGSs, e.g., by the conjugation to cell-penetrating peptides, needs to be optimized before they can be used to target pathogenic bacteria.

\subsubsection{Virulence Genes}

In addition to drug resistance and essential genes, various attempts to target virulence genes by EGSs have been reported [39-41]. It has been shown that Salmonella invasion of host cells can be decreased by the downregulation of $i n v B$ and $i n v C$ mRNA [39]. InvB is a chaperone which is involved in actin rearrangements of the host cell and is not required for invasion; the InvC protein is an ATPase 
required for the type III secretion system involved in the invasion of host cells $[65,66]$. On the Salmonella genome the $i n v B$ and $i n v C$ genes overlap, with the last coding nucleotide of $i n v B$ being the first coding nucleotide of $i n v C$. Therefore, it is likely that $i n v B$ and $i n v C$ are translated from a joint transcript. RNase T1 mapping of single-stranded RNA regions resulted in the design of three EGSs directed to invC and 1 EGS directed to $i n v B$. All of these induced RNase P-mediated cleavage at the predicted site in vitro. For in vivo experiments, both high- and low-copy-number plasmids were generated that allowed the production of two EGSs upon arabinose induction. These two EGSs were either both directed to $i n v B$ or $i n v C$, or one targeted $i n v B$ and the other $i n v C$. All plasmids were capable of decreasing the secretion of SipB and SipC proteins, which are released by the type III secretion system. As expected, the effect was less pronounced for the low-copy number plasmids $(20 \%-30 \%)$ than for the high-copy-number plasmids ( $\geq 65 \%$ ). Interestingly, in the host invasion experiments, the low-copy number plasmids were found to be incapable of reducing invasion in contrast to the high-copy-number plasmids. Since low expression of EGSs reduced type III secretion but did not detectably affect host invasion, the authors hypothesized that a certain level of inhibition of type III secretion has to be reached to inhibit host invasion [39]. In invasion studies, it was shown that a plasmid carrying two EGSs directed to invB also lowers secretion and invasion, which is consistent with a dicistronic invB/invC transcript.

Others have successfully reduced the levels of virulence genes of the pathogenic strains Francisella tularensis and Yersinia pestis [40,41]. In these studies, the virulence genes were expressed in E. coli and down-regulated with multiple EGSs and M1GSs. The F. tularensis virulence gene $m g l B$, required for intracellular growth, was targeted with three different EGSs. After induction of expression of the EGSs, all three were capable of reducing the target mRNA level. On the contrary, three M1GSs that targeted the same sequences did not significantly down-regulate the $m g l B$ mRNA [40]. For $Y$. pestis, three EGSs and three M1GSs targeting the same sequence elements of the $y s c N$ and $Y s c S$ mRNAs were also made. The $y s c N$ and $y s c S$ genes are required to escape from phagocytic killing by the host. The three EGSs all reduced target mRNA expression to $37 \%-68 \%$, whereas only one M1GS significantly reduced the mRNA level.

Taken together, these studies showed that EGSs are effective in reducing pathogenic virulence factor mRNA levels, while the use of M1GSs has been less successful in in vivo studies. Therefore, EGSs targeting virulence genes are promising tools in eliminating pathogenic bacterial infections. However, in the case of $F$. tularensis and $Y$. pestis, the studies have been performed in E. coli and, before they can be used in therapeutic approaches, they need to be tested in the pathogenic strains.

\subsection{Downregulation of Malaria Transcripts and Infections}

The infectious disease malaria is caused by parasites of the genus Plasmodium and is among the most prevalent diseases worldwide. Augagneur and coworkers addressed the question whether RNase P-based modulation of gene expression could be employed to reduce Plasmodium proliferation [31], focusing on the genome of the P. falciparum, which is the most prevalent type of Plasmodium on the African continent. Since $P$. falciparum lacks the RNAi machinery, siRNA approaches targeting the parasitic genes are not possible. Therefore, the EGS methodology might be a feasible approach to target malaria. 
A CPP-PMO EGS that targets the $P$. falciparum gyrA (PfgyrA) gene, which is known to be expressed in all stages of parasite development, was generated. The CPP-PMO EGS was readily detected in infected, but not uninfected erythrocytes, as well as in the parasite. P. falciparum infection leads to increased erythrocyte membrane permeability to a variety of substances and this may facilitate entry of the CPP-PMO EGS in the infected erythrocyte. Subsequently, the CPP-PMO EGS probably enters the parasite via a permease expressed on the parasitic membrane.

The addition of the EGS conjugate to a $P$. falciparum culture synchronized in an early developmental stage resulted in a delay in parasite proliferation, an altered morphology, as well as blocking of early development and replication. This confirmed that the PfgyrA gene seems to have a crucial function in parasite replication and thus is an ideal target. As the CPP-PMO EGSs were equally effective against both drug-sensitive and drug-resistant $P$. falciparum strains, these compounds may not only be used for functional analyses but also in antimalarial therapy. In addition, this technique can be used for the functional analysis of other genes of $P$. falciparum and to establish a list of alternative therapeutic targets [31].

\subsection{Downregulation of Viral RNAs and Infections}

Plehn-Dujowich and colleagues were among the first to use the EGS technology for down-regulation of viral RNAs. RNase T1 mapping was applied to select EGSs against the influenza virus PB2 and $N P$ mRNAs, which encode proteins that are involved in viral replication. They demonstrated that two EGSs targeting $P B 2$ and $N P$ were capable of reducing the expression of the viral M1 protein in infected cells by 70\%-95\%, whereas a single EGS targeting $P B 2$ reduced M1 expression to $42 \%$. This result shows that, like in bacteria, the use of multiple EGSs can be more efficient in inhibiting microbial growth $[35,42]$. Furthermore, they showed that a minimized EGS, lacking the anticodon stem and loop structures, was more effective in guiding target RNA cleavage in vitro as well as in vivo compared to the tRNA-derived EGS.

In another early application of the EGS technology the infectious herpes simplex virus 1 (HSV1) thymidine kinase (TK) mRNA was targeted. Liu and coworkers analyzed the TK mRNA structure using in vivo DMS mapping. A region near the translation initiation site was found to be the most accessible part of the mRNA. Stable expression of a minimized EGS targeting this region reduced the TK mRNA level by $80 \%$ in human cell lines infected with HSV1, whereas a tRNA-derived EGS reduced the mRNA level up to 50\% [44]. Later the minimized EGS was further optimized by an in vitro selection procedure of partially randomized sequences. The resulting EGS was capable of decreasing the TK mRNA levels up to $96 \%$ in infected cells [45]. In another approach, the HSV1 ICP4 mRNA was targeted using a M1GS [43]. The ICP4 protein is a transcription activator required for expression of the viral early and late genes [67]. Stable expression of the M1GS significantly reduced the levels of ICP4 mRNA $(80 \%)$ and protein $(87 \%)$ as well as that of some early and late viral genes in virus infected cells. In addition, a 1000-fold reduction of viral growth was observed.

\subsubsection{Cytomegalovirus}

Human cytomegalovirus (HCMV) is a common herpes virus, which usually affects newborns and adults with compromised immune systems, causing significant morbidity and mortality [68]. Various 
efforts have been made to target and inhibit HCMV using both EGSs and M1GSs [32-34,47-55,69-71]. The IE1 and IE2 immediate early proteins are transcription factors involved in the activation of viral gene expression. Multiple examples are reported in which IE1 and IE2 have been targeted to inhibit HCMV proliferation [47-49,69]. IE1 and IE2 are encoded by the same gene and result from alternative splicing of the pre-mRNA. Liu and colleagues have used an M1GS to target the overlapping regions of the IE1 and IE2 mRNAs corresponding to a shared exon. A significant decrease in IE1/2 mRNA and protein levels, as well as the levels of some viral early and late proteins, was observed. Moreover, viral growth was inhibited 150-fold [69]. Later, two M1GS variants, which carry M1 point mutations that enhance the M1 catalytic activity, have been used to target the same region on the IE1 and IE2 mRNAs [47,48]. These variants increased viral growth inhibition compared to the wild type (WT) M1GS, leading to a 3000- and 10,000-fold reduction. In another study, an M1GS variant specifically targeting the IE2 mRNA inhibited viral growth 3500-fold [49].

Capsid assembly protein (AP) and protease (PR) mRNAs have also been extensively used as targets to inhibit HCMV growth. AP and PR are essential for viral encapsulation and have an overlapping region on their mRNAs. A minimized EGS specifically targeting the PR mRNA reduced viral growth 500-fold, whereas a WT M1GS targeting the same region seemed less effective since it reduced viral growth 100-fold [50,53]. Other minimized EGSs targeting both PR and AP have led to 800- and even 7000-fold inhibition of viral growth [51,52], whereas a WT M1GS targeting both AP and PR mRNA inhibited viral growth up to 2000-fold [54]. Very recently, an M1GS variant, carrying two M1 point mutations, was used to inhibit HCMV replication. Expression of the M1GS variant in stable human cell lines decreased the target mRNA and protein expression almost completely (99\%), whereas the WT M1GS decreased the target mRNA and protein levels to 75\% (mRNA determined $48 \mathrm{~h}$ and protein $72 \mathrm{~h}$ after infection). More importantly, the M1GS variant was capable to reduce viral titers 50,000-fold [55].

As discussed in the previous section, attenuated Salmonella-based delivery may have important advantages for therapeutic applications of guide sequences. For mouse CMV (MCMV) targeting, two strains (SL101 and SL201) were tested in cultured macrophages and in severe combined immune deficiency (SCID) mice. These strains were derived from the attenuated SL7202 strain, which is defective in aromatic amino acid biosynthesis, and in addition SL101 has a deletion of ssrA/B genes and SL201 a deletion in the $m s b B$ gene $[33,34]$. The ssrA/B and msbB genes are involved in virulence as well as pathogenesis of Salmonella. SL101 was used for the delivery of an M1GS construct against AP and PR. MCMV-infected macrophages treated with the SL101 Salmonella M1GS showed 80\%-85\% reduction of target protein expression and 2500-fold reduction in viral growth. Orally administered SL101 Salmonella M1GS in SCID mice resulted in efficient delivery to spleen and liver, as well as reduced viral gene expression, decreased viral titers, and improved survival [33]. Salmonella SL201 was used for the delivery of an EGS construct against MCMV PR. Treated macrophages showed a reduction of $86 \%$ in protein expression and 3000 -fold reduced viral titers. In MCMV infected mice, a decrease in viral gene expression was observed, as well as viral growth reduction in various organs, and increased survival [34].

Even though Salmonella-based delivery of M1GSs and EGSs provides a promising approach for combatting viral infections, these Salmonella strains are not capable of completely eradicating the pathogens in the SCID mice, even after repeated inoculations with the M1GS and EGS strains. The reasons for this are unclear, since no research has been done to address this question. A possible 
explanation might be that the RNAs are not stable and rapidly degraded in the host cells. Alternatively, the effectiveness of the M1GS and EGS might not be sufficient to degrade all target RNAs in the host cell. Indeed, when using HCMV-infected J774 cells the SL101 M1GS and SL201 EGS did not completely eliminate the target RNAs $[33,34]$. However, in the SCID mice, the levels of target RNA were barely detectable 14 days after infection. Because M1GS variants with increased catalytic activity have been shown to be very effective in cell assays, it would be interesting to see whether Salmonella-based delivery of these variants could further prolong the survival of infected mice, or even completely eradicate the virus.

\subsubsection{Hepatitis B Virus}

Hepatitis B virus (HBV) chronically infects over 400 million individuals worldwide [72]. HBV leads to a persistent and chronic life-long infection in hepatocytes, which can cause cirrhosis, liver failure, and liver cancer [73]. Delivery of therapeutic drugs specifically into the liver is a challenge. Since Salmonella can easily infect hepatocytes, Salmonella might serve as a vehicle for EGS delivery to the liver. Indeed, Lu and coworkers [57] have introduced a minimized EGS with the SL201 Salmonella strain into mammalian cells. The EGS was directed towards the HBV pre-genomic RNA (pgRNA) and the pre-S/L and S mRNAs. The pgRNA has a dual function as the template for viral genome synthesis and as mRNA encoding the viral polymerase and core protein. The selected target sequence also overlaps with the pre-S/L and S mRNAs, encoding viral surface antigens. The Salmonella delivered EGS decreased capsid associated HBV RNA 6000-fold in mammalian cells [57]. Liu and coworkers [56] used the SL7202-derived Salmonella strain SL301 to deliver a minimized EGS to liver cells. In the SL301 strain, the spiR gene, important for Salmonella intracellular survival and virulence, has been deleted. SL301 did not show virulence or toxicity in vivo as all mice were alive 85 days after inoculation. By DMS mapping experiments, the accessibility of the pgRNA in human hepatoma cells with a stable HBV infection (HepG2.2.15) was determined. Salmonella SL301-mediated delivery of the EGS was tested in cultured liver cells (HepG2) as well as in mice and in both cases gene transfer was successful; after 24 h, more than $70 \%$ of the HepG2 cells were infected with the SL301 carrying the EGS (SL301-EGS) and in SCID mice, a substantial amount of SL301-EGS infected cells was detected in the liver and spleen 10 days after intragastric inoculation. In HepG2.2.15, containing the full-length genome of HBV, more than $90 \%$ reduction of HBV mRNA and antigens was observed $48 \mathrm{~h}$ after infection with the SL301-EGS. Moreover, SL301-EGS infection led to a 2000-fold reduction in intracellular capsid-associated HBV DNA, measured $72 \mathrm{~h}$ after the infection. HBV cannot infect murine hepatocytes due to the lack of HBV-receptors, therefore, mice liver cells were transfected with the HBV plasmid using an intravenous hydrodynamic injection. Five days after oral inoculation of Salmonella, the pgRNA and pre-S/L transcripts in the liver were reduced by $95 \%$. HBV core antigen was barely detectable in liver sections of treated mice, whereas a substantial amount of this protein was present in non-treated mice. In addition, a 95\% reduction of HBV gene expression and a 200,000-fold reduction in viral DNA levels were seen in the livers and sera of mice upon treatment [56]. These results indicates that the endogenous RNase $\mathrm{P}$ is highly efficient and specifically cleaves target RNA, and that mutated Salmonella strains result in efficient delivery of the EGS transcript in the infected cells. 


\subsubsection{Hepatitis C Virus}

Hepatitis $\mathrm{C}$ virus (HCV) infects nearly $3 \%$ of the population worldwide and is a major cause of liver disease resulting in persistent acute and chronic infections, which can, similar to HBV, give rise to liver damage $[73,74]$. Currently, no vaccine is available to prevent HCV infection. The single-stranded RNA genome of HCV comprises a relatively conserved 5'-untranslated region, which contains an internal ribosome entry site (IRES) that is important for the initiation of viral polyprotein translation. Using computational secondary structure prediction, the position 67 nucleotides downstream from the first nucleotide of the HCV genomic RNA was chosen as the M1GS cleavage site, as it seemed to be in one of the most accessible regions and contains flanking sequences needed for proper M1GS interaction. Two M1GS constructs were made, M1GS-HCV/C67 with a linker sequence between the M1 RNA and the 13-nucleotide GS, and M1GS-HCV/C67* without a linker sequence. In vitro, the linker sequence appeared to be required for efficient cleavage of the genomic RNA of HCV [58]. To improve the pharmacokinetic properties of M1GS-HCV/C67, cholesterol was conjugated to its 5'-end, which did not diminish the cleavage activity. Both M1GS-HCV/C67 and Cholesterol-M1GS-HCV/C67 resulted in an $85 \%$ reduction in $\mathrm{HCV}$ core protein expression, as well as a more than 1000 -fold reduction of viral growth in transfected Huh7.5.1 cells. These results indicate that the M1GS approach allows the efficient inhibition of HCV growth, that a linker between the M1 and GS parts may be crucial for activity, and that the cholesterol modification does not reduce its antiviral activity and therefore might be used to improve the kinetics for M1GS ribozymes [58].

\subsection{Downregulation of Human Genes to Target HIVI}

Human Immunodeficiency Virus (HIV) is the etiological agent of Acquired Immunodeficiency Syndrome (AIDS) [75,76]. The first study on the use of EGSs targeting HIV demonstrated that EGSs targeting tat mRNA and long terminal repeat (LTR) RNA can successfully inhibit HIV replication in COS cells [59]. Later, Liu and coworkers developed an M1GS-based approach against HIV1, one of the two major types of HIV, by targeting the HIV tat mRNA [60]. An M1GS variant, with two mutations (G83U and G340A) led to 90\% reduction of viral RNA expression and a 150-fold reduction in viral growth. More recently the same researchers took a different approach by targeting the mRNA encoding the human CC-chemokine receptor 5 (CCR5) [61]. This receptor is the primary co-receptor for HIV1 to establish an initial infection and therefore an attractive host target gene to interfere with HIV1 infection. CCR5 belongs to the $\beta$ subfamily of chemokine receptors and due to functional similarities within this family CCR5 was not expected to be essential for the human cell. Because a host gene is targeted, counteracting retroviral mutations are expected to occur less easily. In addition, reducing CCR5 affects the initial step of HIV1 infection before genome integration and, therefore, may prevent mutations which can cause resistance. Moreover, a naturally occurring 32 base-pair deletion in the CCR5 gene causes partial resistance towards HIV1 and therefore CCR5 was considered to be an ideal target for anti-HIV therapy.

The accessibility of the region around the translation initiation site of CCR 5 mRNA was determined by in vivo DMS mapping and a position 29 nucleotides downstream from the translation initiation codon was chosen as cleavage site for human RNase P in the design of a minimized EGS. The CCR5 
mRNA specificity of the EGS was substantiated in additional experiments. No differences in growth and viability were observed between the vector with or without EGS, indicating that the EGS does not cause significant cytotoxicity. In PM1 cells (a HIV1-permissive T-cell clone), more than $70 \%$ reduction in CCR5 mRNA and protein expression was observed, whereas control EGSs only led to minor reduction in these levels. To test the specificity and the ability of the EGS to prevent HIV infection, two HIV strains were used, the HIVBa-L strain, which depends on the CCR5 co-receptor for infection, and the HIVIIIB strain, which depends on the CXCR4 co-receptor for host infection. The EGS led to a 50-fold inhibition of HIVBa-L infection, and did not detectably affect HIVIIIB infection. Altogether, these data showed that HIV replication is inhibited by the EGS with a very high specificity. However, because 50-fold inhibition may be insufficient in completely blocking HIV infections, for therapeutic applications more efficient EGSs are required and the EGS might be combined with RNAi to enhance the efficiency of the approach [61].

\subsection{Downregulation of the BCR-ABL Oncogenic Transcript}

The Philadelphia chromosome, resulting from a translocation between chromosomes 9 and 22, is found in patients with acute lymphoblastic leukemia and chronic myelogenous leukemia. During translocation the $A B L$ gene, located on chromosome 22, and the $B C R$ gene, located on chromosome 9, are fused together $[77,78]$. The breakpoint within the $B C R$ gene can be either in exon 1 or exon 3 leading to BCR-ABL fusion proteins of $190 \mathrm{kDa}(\mathrm{p} 190)$ and $210 \mathrm{kDa}(\mathrm{p} 210)$, respectively. These fusion proteins play a role in cancer etiology by inducing Bcl-2 mediated inhibition of apoptosis [79]. Because the chimeric mRNA resulting from the translocation is unique to the cancer cells and plays a role in cancer etiology, it is a promising target for therapeutic agents. Cobaleda and Garcia have used the M1GS approach to specifically target the chimeric mRNAs by using guide sequences targeting the $B C R / A B L$ junctions [62]. They used murine $\mathrm{Ba} / \mathrm{F} 3$ cell lines expressing either p190 or p210 to demonstrate the effectiveness and specificity of M1GSs. An M1GS targeting p190 specifically cleaved the p190 mRNA and not the p210 mRNA and vice versa. Moreover, treatment with the M1GS in the corresponding cell lines led to reduction of $B c l-2$ mRNA levels and enhanced cell death. In these experiments, expression of the M1GSs was achieved by stably transducing cells with a retroviral vector via electroporation. Since this delivery is not suitable for therapeutic applications, alternative delivery systems need to be explored.

\subsection{Prophylactic vs. Therapeutic Treatment with EGS/MIGS}

In the case of the bacterial targets, most of the experiments in which the effects of EGS/M1GS were assessed were performed with cultured bacteria and therefore can be considered drug-like approaches. In addition, the targeting of $S$. aureus in the murine cutaneous wound model was initiated after the infection of the wounds with the bacteria. To study the effects of EGSs on invasion of cells by Salmonella, EGS expression was induced in the bacteria prior to the incubation of the Henle-407 cells with the Salmonella strains.

The experiments that were aimed at EGS/M1GS-mediated interference of the proliferation of viruses were almost all performed in stably transfected cultured cells that expressed the EGS/M1GS prior to infection with the viruses. In some studies, the EGSs were internalized by liposome-mediated 
transfection procedures, but also in these cases this step preceded the infection of the cells with viruses. In spite of the prophylactic anti-viral efficacy of the EGSs and M1GSs, these molecules also appeared to display anti-viral activities in therapeutic settings. This was demonstrated in the studies with Salmonella-mediated delivery of the EGSs, both in cell culture and in mice. In the latter, intraperitoneal infection with the virus, for example, preceded repeated oral inoculation with the EGS-containing Salmonella strains. Taken together, these data strongly suggest that the EGS/M1GS approach can be applied both prophylactically and therapeutically.

\section{EGS/M1GS Approaches vs. RNA Interference}

The use of short RNA interference (RNAi) has also been extensively studied in eradicating microbial infections, cancer and other diseases. Currently, multiple siRNAs (small interfering RNAs) have already been or are being used in clinical trials [80]. Although EGSs and M1GSs have been successfully applied in cell cultures and mice to reduce microbial growth, to our knowledge, no clinical trials with these RNAs have been performed yet. In view of the therapeutic applicability of EGSs/M1GSs and RNAi, it would be interesting to know the difference in, for example, efficacy and potency of RNAi and EGSs/M1GSs targeting the same RNAs. However, limited experimental data is available that directly compare the use of siRNAs and EGSs [81,82]. Hayday and coworkers have targeted the thymosin beta 4 (Tß4) gene with both short hairpin RNAs (shRNAs, which are functionally related to siRNAs) and EGSs. The results demonstrated that the efficacy of the EGS-mediated downregulation of T $\beta 4$ mRNA was much lower ( $0 \%-15 \%)$ compared to that of shRNAs (85\%-90\%) [82]. In contrast, a comparison of EGSs and siRNAs targeting RNase P protein mRNAs showed that EGSs and RNAi can induce similar levels of target down-regulation [81]. Moreover, when using EGSs, a significant reduction in protein levels was already observed after $24 \mathrm{~h}$, whereas it took 48 to $96 \mathrm{~h}$ before the effect of siRNAs became evident. It should be noted that, in both studies, tRNA-derived EGSs have been used, whereas it has been demonstrated that minimized EGSs or M1GSs can induce RNase P cleavage more efficiently. It is clear that more research is required to gain insight in the difference in efficacies of RNAi and RNase P-mediated downregulation of RNAs.

\section{Conclusions}

The RNase P EGS and M1GS technologies to knockdown specific RNAs have been shown to be a useful strategy in combating infectious diseases. EGSs and M1GSs successfully reduced bacterial viability, malaria replication and viral infections in cell cultures, and, to a lesser extent, in multicellular organisms. In general, the effectiveness still needs to be substantiated in infected organisms and also toxic side-effects need to be investigated further. Salmonella-based oral delivery of EGS and M1GS appears to be an attractive way to combat some viral infections in mice and possibly other organisms. Further improvements can be achieved by the introduction of stabilizing moieties and the conjugation of cell-penetrating peptides. CPP-PMOs, for example, were successfully applied in Staphylococcus aureus wound infections, where they improved wound healing. Although various efforts have been made to improve the targeted delivery of EGS/M1GS molecules, the delivery remains a major challenge for therapeutic applications. 


\section{Acknowledgments}

We apologize to the authors whose relevant work we missed citing in this review.

\section{Author Contributions}

Merel Derksen and Vicky Mertens collected the literature that formed the basis for this article. All authors contributed to the writing of this article.

\section{Conflicts of Interest}

The authors declare no conflict of interest.

\section{References}

1. Robertson, H.D.; Altman, S.; Smith, J.D. Purification and properties of a specific Escheria coli ribonuclease which cleaves a tyrosine transfer riboncleic acid precursor. J. Biol. Chem. 1972, 274, 5243-5251.

2. Hernandez-Cid, A.; Aguirre-Sampieri, S.; Diaz-Vilchis, A.; Torres-Larios, A. Ribonucleases P/MRP and the expanding ribonucleoprotein world. Int. Union Biochem. Mol. Biol. Life 2012, 64, 521-528.

3. Guerrier-Takada, C.; Gardiner, K.; Marsh, T.; Pace, N.; Altman, S. The RNA moiety of ribonuclease P is the catalytic subunit of the enzyme. Cell 1983, 35, 849-857.

4. Pannucci, J.A.; Haas, E.S.; Hall, T.A.; Harris, J.K.; Brown, J.W. RNase P RNAs from some Archaea are catalytically active. Proc. Natl. Acad. Sci. USA 1999, 96, 7803-7808.

5. Kikovska, E.; Svärd, S.G.; Kirsebom, L.A. Eukaryotic RNase P RNA mediates cleavage in the absence of protein. Proc. Natl. Acad. Sci. USA 2007, 104, 2062-2067.

6. Holzmann, J.; Frank, P.; Löffler, E.; Bennett, K.L.; Gerner, C.; Rossmanith, W. RNase P without RNA: Identification and functional reconstitution of the human mitochondrial tRNA processing enzyme. Cell 2008, 135, 462-474.

7. Gobert, A.; Gutmann, B.; Taschner, A.; Gössringer, M.; Holzmann, J.; Hartmann, R.K.; Rossmanith, W.; Giegé, P. A single Arabidopsis organellar protein has RNase P activity. Nat. Struct. Mol. Biol. 2010, 17, 740-744.

8. Gutmann, B.; Gobert, A.; Giegé, P. PRORP proteins support RNase P activity in both organelles and the nucleus in Arabidopsis. Genes Dev. 2012, 26, 1022-1027.

9. Taschner, A.; Weber, C.; Buzet, A.; Hartmann, R.K.; Hartig, A.; Rossmanith, W. Nuclear RNase P of Trypanosoma brucei: A single protein in place of the multicomponent RNA-protein complex. Cell Rep. 2012, 2, 19-25.

10. Sugita, C.; Komura, Y.; Tanaka, K.; Kometani, K.; Satoh, H.; Sugita, M. Molecular characterization of three PRORP proteins in the moss Physcomitrella patens: nuclear PRORP protein is not essential for moss viability. PLOS ONE 2014, 9, e108962.

11. Weber, C.; Hartig, A.; Hartmann, R.K.; Rossmanith, W. Playing RNase P evolution: Swapping the RNA catalyst for a protein reveals functional uniformity of highly divergent enzyme forms. PLoS Genet. 2014, 10, e1004506. 
12. McClain, W.H.; Guerrier-Takada, C.; Altman, S. Model substrates for an RNA enzyme. Science 1987, 238, 527-530.

13. Forster, A.C.; Altman, S. External Guide Sequences for an RNA molecule. Science 1990, 249, $783-786$.

14. Li, Y.; Guerrier-Takada, C.; Altman, S. Targeted cleavage of mRNA in vitro by RNase P from Escherichia coli. Proc. Natl. Acad. Sci. USA 1992, 89, 3185-3189.

15. Yuan, Y.; Hwang, E.S.; Altman, S. Targeted cleavage of mRNA by human RNase P. Proc. Natl. Acad. Sci. USA 1992, 89, 8006-8010.

16. Raj, M.L.; Pulukkunat, D.K.; Reckard, J.F.; Thomas, G.; Gopalan, V. Cleavage of bipartite substrates by rice and maize ribonuclease P. Application to degradation of target mRNAs in plants. Plant Physiol. 2001, 125, 1187-1190.

17. Rangarajan, S.; Raj, M.L.S.; Hernandez, J.M.; Grotewold, E.; Gopalan, V. RNase P as a tool for disruption of gene expression in maize cells. Biochem. J. 2004, 380, 611-616.

18. Guerrier-Takada, C.; Li, Y.; Altman, S. Artificial regulation of gene expression in Escherichia coli by RNase P. Proc. Natl. Acad. Sci. USA 1995, 92, 11115-11119.

19. Wegscheid, B.; Hartmann, R.K. The precursor tRNA 3'-CCA interaction with Escherichia coli RNase P RNA is essential for catalysis by RNase $\mathrm{P}$ in vivo. RNA 2006, 12, 2135-2148.

20. Yuan, Y.; Altman, S. Selection of guide sequences that direct efficient cleavage of mRNA by human ribonuclease P. Science 1994, 263, 1269-1273.

21. Yuan, Y.; Altman, S. Substrate recognition by human RNase P: Identification of small, model substrates for the enzyme. EMBO J. 1995, 14, 159-168.

22. Ma, M.; Benimetskaya, L.; Lebedeva, I.; Dignam, J.; Takle, G.; Stein, C.A. Intracellular mRNA cleavage induced through activation of RNase P by nuclease-resistant external guide sequences. Nat. Biotechnol. 2000, 18, 58-61.

23. Liu, F.; Altman, S. Inhibition of viral gene expression by the catalytic RNA subunit of RNase P from Escherichia coli. Genes Dev. 1995, 9, 471-480.

24. Li, Y.; Altman, S. Cleavage by RNase P of gene N mRNA reduces bacteriophage $\lambda$ burst size. Nucleic Acids Res. 1996, 24, 835-842.

25. Ma, M.Y.; Jacob-samuel, B.U.I.; Dignam, J.C.; Pace, U.; Goldberg, A.R.; George, S.T. Nuclease-resistanct external guide sequence-induced cleavage of target RNA by human ribonuclease P. Antisense Nucleic Acid Drug Dev. 1998, 8, 415-426.

26. Hudziak, R.M.; Barofsky, E.; Barofsky, D.F.; Weller, D.L.; Huang, S.B.; Weller, D.D. Resistance of morpholino phosphodiamidate oligomers to enzymatic degradation. Antisense Nucleic Acid Drug Dev. 1996, 6, 267-272.

27. Soler Bistué, A.J.C.; Martín, F.A.; Vozza, N.; Ha, H.; Joaquín, J.C.; Zorreguieta, A.; Tolmasky, M.E. Inhibition of $\mathrm{AAC}\left(6^{\prime}\right)$-Ib-mediated amikacin resistance by nuclease-resistant external guide sequences in bacteria. Proc. Natl. Acad. Sci. USA 2009, 106, 13230-13235.

28. Shen, N.; Ko, J.; Xiao, G.; Wesolowski, D.; Shan, G.; Geller, B.; Izadjoo, M.; Altman, S. Inactivation of expression of several genes in a variety of bacterial species by EGS technology. Proc. Natl. Acad. Sci. USA 2009, 106, 8163-8168. 
29. Wesolowski, D.; Alonso, D.; Altman, S. Combined effect of a peptide-morpholino oligonucleotide conjugate and a cell-penetrating peptide as an antibiotic. Proc. Natl. Acad. Sci. USA 2013, 110, 8686-8689.

30. Wesolowski, D.; Tae, H.S.; Gandotra, N.; Llopis, P.; Shen, N.; Altman, S. Basic peptide-morpholino oligomer conjugate that is very effective in killing bacteria by gene-specific and nonspecific modes. Proc. Natl. Acad. Sci. USA 2011, 108, 16582-16587.

31. Augagneur, Y.; Wesolowski, D.; Tae, H.S.; Altman, S.; Ben Mamoun, C. Gene selective mRNA cleavage inhibits the development of Plasmodium falciparum. Proc. Natl. Acad. Sci. USA 2012, 109, 6235-6240.

32. Bai, Y.; Li, H.; Vu, G.P.; Gong, H.; Umamoto, S.; Zhou, T.; Lu, S.; Liu, F. Salmonella-mediated delivery of RNase P-based ribozymes for inhibition of viral gene expression and replication in human cells. Proc. Natl. Acad. Sci. USA 2010, 107, 7269-7274.

33. Bai, Y.; Gong, H.; Li, H.; Vu, G.P.; Lu, S.; Liu, F. Oral delivery of RNase P ribozymes by Salmonella inhibits viral infection in mice. Proc. Natl. Acad. Sci. USA 2011, 108, 3222-3227.

34. Jiang, X.; Gong, H.; Chen, Y.C.; Vu, G.P.; Trang, P.; Zhang, C.Y.; Lu, S.; Liu, F. Effective inhibition of cytomegalovirus infection by external guide sequences in mice. Proc. Natl. Acad. Sci. USA 2012, 109, 13070-13075.

35. Guerrier-Takada, C.; Salavati, R.; Altman, S. Phenotypic conversion of drug-resistant bacteria to drug sensitivity. Proc. Natl. Acad. Sci. USA 1997, 94, 8468-8472.

36. McKinney, J.; Guerrier-Takada, C.; Wesolowski, D.; Altman, S. Inhibition of Escherichia coli viability by external guide sequences complementary to two essential genes. Proc. Natl. Acad. Sci. USA 2001, 98, 6605-6610.

37. Sala, C.D.; Soler-Bistué, A.J.C.; Korprapun, L.; Zorreguieta, A.; Tolmasky, M.E. Inhibition of cell division induced by external guide sequences (EGS Technology) targeting ftsZ. PLoS ONE 2012, 7, 1-7.

38. Sawyer, A.J.; Wesolowski, D.; Gandotra, N.; Stojadinovic, A.; Izadjoo, M.; Altman, S.; Kyriakides, T.R. A peptide-morpholino oligomer conjugate targeting Staphylococcus aureus gyrA mRNA improves healing in an infected mouse cutaneous wound model. Int. J. Pharm. 2013, 453, $651-655$.

39. McKinney, J.S.; Zhang, H.; Kubori, T.; Galán, J.E.; Altman, S. Disruption of type III secretion in Salmonella enterica serovar Typhimurium by external guide sequences. Nucleic Acids Res. 2004, $32,848-854$.

40. Xiao, G.; Lundblad, E.W.; Izadjoo, M.; Altman, S. Inhibition of expression in Escherichia coli of a virulence regulator $\mathrm{MglB}$ of Francisella tularensis using external guide sequence technology. PLOS ONE 2008, 3, 1-7.

41. Ko, J.; Izadjoo, M.; Altman, S. Inhibition of expression of virulence genes of Yersinia pestis in Escherichia coli by external guide sequences and RNase P. RNA 2008, 14, 1656-1662.

42. Plehn-Dujowich, D.; Altman, S. Effective inhibition of influenza virus production in cultured cells by external guide sequences and ribonuclease P. Proc. Natl. Acad. Sci. USA 1998, 95, 7327-7332.

43. Trang, P.; Kilani, A.; Kim, J.; Liu, F. A ribozyme derived from the catalytic subunit of RNase P from Escherichia coli is highly effective in inhibiting replication of herpes simplex virus 1 . J. Mol. Biol. 2000, 301, 817-826. 
44. Kawa, D.; Wang, J.; Yuan, Y.; Liu, F. Inhibition of viral gene expression by human ribonuclease P. RNA 1998, 4, 1397-1406.

45. Zhou, T.; Kim, J.; Kilani, A.F.; Kim, K.; Dunn, W.; Jo, S.; Nepomuceno, E.; Liu, F. In vitro selection of external guide sequences for directing RNase P-mediated inhibition of viral gene expression. J. Biol. Chem. 2002, 277, 30112-30120.

46. Trang, P.; Kilani, A.; Lee, J.; Hsu, A.; Liou, K.; Kim, J.; Nassi, A.; Kim, K.; Liu, F. RNase P ribozymes for the studies and treatment of human cytomegalo virus infections. J. Clin. Virol. 2002, 25, S63-S74.

47. Trang, P.; Hsu, A.; Zhou, T.; Lee, J.; Kilani, A.F.; Nepomuceno, E.; Liu, F. Engineered RNase P ribozymes inhibit gene expression and growth of cytomegalovirus by increasing rate of cleavage and substrate binding. J. Mol. Biol. 2002, 315, 573-586.

48. Zou, H.; Lee, J.; Umamoto, S.; Kilani, A.F.; Kim, J.; Trang, P.; Zhou, T.; Liu, F. Engineered RNase $\mathrm{P}$ ribozymes are efficient in cleaving a human cytomegalovirus mRNA in vitro and are effective in inhibiting viral gene expression and growth in human cells. J. Biol. Chem. 2003, 278, 37265-37274.

49. Yang, Z.; Vu, G.P.; Qian, H.; Chen, Y.C.; Wang, Y.; Reeves, M.; Zen, K.; Liu, F. Engineered RNase P ribozymes effectively inhibit human cytomegalovirus gene expression and replication. Viruses 2014, 6, 2376-2391.

50. Dunn, W.; Trang, P.; Khan, U.; Zhu, J.; Liu, F. RNase P-mediated inhibition of cytomegalovirus protease expression and viral DNA encapsidation by oligonucleotide external guide sequences. Proc. Natl. Acad. Sci. USA 2001, 98, 14831-14836.

51. Li, H.; Trang, P.; Kim, K.; Zhou, T.; Umamoto, S.; Liu, F. Effective inhibition of human cytomegalovirus gene expression and growth by intracellular expression of external guide sequence RNA. RNA 2006, 12, 63-72.

52. Jiang, X.; Chen, Y.C.; Gong, H.; Trang, P.; Lu, S.; Liu, F. Ribonuclease P-mediated inhibition of human cytomegalovirus gene expression and replication induced by engineered external guide sequences. RNA Biol. 2012, 9, 1186-1195.

53. Trang, P.; Kim, K.; Zhu, J.; Liu, F. Expression of an RNase P ribozyme against the mRNA encoding human cytomegalovirus protease inhibits viral capsid protein processing and growth. J. Mol. Biol. 2003, 328, 1123-1135.

54. Kim, K.; Umamoto, S.; Trang, P.; Hai, R.; Liu, F.Y. Intracellular expression of engineered RNase P ribozymes effectively blocks gene expression and replication of human cytomegalovirus. RNA 2004, 10, 438-447.

55. Yang, Z.; Reeves, M.; Ye, J.; Trang, P.; Zhu, L.; Sheng, J.; Wang, Y.; Zen, K.; Wu, J.; Liu, F. RNase P ribozymes inhibit the replication of human cytomegalovirus by targeting essential viral capsid proteins. Viruses 2015, 7, 3345-3360.

56. Xia, C.; Chen, Y.C.; Gong, H.; Zeng, W.; Vu, G.P.; Trang, P.; Lu, S.; Wu, J.; Liu, F. Inhibition of hepatitis B virus gene expression and replication by ribonuclease P. Mol. Ther. 2013, 21, 995-1003.

57. Zhang, Z.; Vu, G.P.; Gong, H.; Xia, C.; Chen, Y.C.; Liu, F.; Wu, J.; Lu, S. Engineered external guide sequences are highly effective in inhibiting gene expression and replication of hepatitis $\mathrm{B}$ virus in cultured cells. PLOS ONE 2013, 8, e65268. 
58. Mao, X.; Li, X.; Mao, X.; Huang, Z.; Zhang, C.; Zhang, W.; Wu, J.; Li, G. Inhibition of hepatitis C virus by an M1GS ribozyme derived from the catalytic RNA subunit of Escherichia coli RNase P. Virol. J. 2014, doi:10.1186/1743-422X-11-86.

59. Barnor, J.S.; Endo, Y.; Habu, Y.; Miyano-Kurosaki, N.; Kitano, M.; Yamamoto, H.; Takaku, H. Effective inhibition of HIV-1 replication in cultured cells by external guide sequences and ribonuclease P. Bioorg. Med. Chem. Lett. 2004, 14, 4941-4944.

60. Zeng, W.; Chen, Y.C.; Bai, Y.; Trang, P.; Vu, G.P.; Lu, S.; Wu, J.; Liu, F. Effective inhibition of human immunodeficiency virus 1 replication by engineered RNase P ribozyme. PLoS ONE 2012, 7, e51855.

61. Zeng, W.; Vu, G.P.; Bai, Y.; Chen, Y.C.; Trang, P.; Lu, S.; Xiao, G.; Liu, F. RNase P-associated external guide sequence effectively reduces the expression of human CC-chemokine receptor 5 and inhibits the infection of human immunodeficiency virus 1. BioMed Res. Int. 2013, doi:10.1155/ 2013/509714.

62. Cobaleda, C.; Sánchez-García, I. In vivo inhibition by a site-specific catalytic RNA subunit of RNase P designed against the BCR-ABL oncogenic products: A novel approach for cancer treatment. Blood 2000, 95, 731-737.

63. Tolmasky, M.E. Aminoglycoside-modifying enzymes: Characteristics, localization, and dissemination. In Enzyme-Mediated Resistance to Antibiotics; Bonomo, R., Tolmask, A., Eds.; ASM Press: Washington, DC, USA, 2007; pp. 35-52.

64. Soler Bistué, A.J.C.; Ha, H.; Sarno, R.; Don, M.; Zorreguieta, A.; Tolmasky, M.E. External guide sequences targeting the $A A C\left(6^{\prime}\right)-I b$ mRNA induce inhibition of amikacin resistance. Antimicrob. Agents Chemother. 2007, 51, 1918-1925.

65. Eichelberg, K.; Ginocchio, C.C.; Galan, J.E. Molecular and functional characterization of the Salmonella typhimurium invasion genes $i n v B$ and $i n v C$ : Homology of invC to the F0F1 ATPase family of proteins. J. Bacteriol. 1994, 176, 4501-4510.

66. Bronstein, P.A.; Miao, E.A. InvB is a type III secretion chaperone specific for SspA. J. Bacteriol. 2000, 182, 6638-6644.

67. De Luca, N.A.; McCarthy, A.M.; Schaffer, P.A. Isolation and characterization of deletion mutants of herpes simplex virus type 1 in the gene encoding immediate-early regulatory protein ICP4. J. Virol. 1985, 56, 558-570.

68. Mocarski, E.S.; Shenk, T.; Pass, R. Cytomegalovirus. In Fields Virology; Knipe, D., Howley, P., Eds.; Lippincott Williams and Wilkins: Philadelphia, PA, USA, 2007; pp. 2701-2772.

69. Trang, P.; Lee, M.; Nepomuceno, E.; Kim, J.; Zhu, H.; Liu, F. Effective inhibition of human cytomegalovirus gene expression and replication by a ribozyme derived from the catalytic RNA subunit of RNase P from Escherichia coli. Proc. Natl. Acad. Sci. USA 2000, 97, 5812-5817.

70. Zeng, Z.; Li, H.; Li, Y.; Cui, Y.; Zhou, Q.; Yang, G.; Zhou, T. Effective inhibition of human cytomegalovirus gene expression by DNA-based external guide sequences. Acta Biochim. Biophys. Sin. 2009, 41, 389-398.

71. Zhang, W.; Li, H.; Li, Y.; Zeng, Z.; Li, S.; Zhang, X.; Zou, Y.; Zhou, T. Effective inhibition of HCMV UL49 gene expression and viral replication by oligonucleotide external guide sequences and RNase P. Virol. J. 2010, doi:10.1186/1743-422X-7-100. 
72. Seegar, C.; Zoulim, F.; Mason, W. Hepadnaviruses. In Fields Virology; Knipe, D., Howley, P., Eds.; Lippincott Willam and Wilkins: Philadelphia, PA, USA, 2007; pp. 2978-3029.

73. Guidotti, L.G.; Chisari, F.V. Immunobiology and pathogeneis of viral hepatitis. Annu. Rev. Pathol. 2006, 1, 23-61.

74. Tang, H.; Grisé, H. Cellular and molecular biology of HCV infection and hepatitis. Clin. Sci. 2006, 117, 49-65.

75. Freed, E.; Martin, M. HIVs and their replication. In Fields Virology; Knipe, D., Howley, P., Eds.; Lippincott Williams and Wilkins: Philadelphia, PA, USA, 2007; pp. 2108-2185.

76. Kurtizkes, D.; Walker, B. HIV-1: Pathogenesis, clinical manifestations, and treatment. In Fields Virology; Knipe, D., Howley, P., Eds.; Lippincott Willam and Wilkins: Philadelphia, PA, USA, 2007; pp. 2188-2214.

77. Hermans, A.; Heisterkamp, N.; von Linden, M.; van Baal, S.; Meijer, D.; van der Plas, D.; Wiedemann, L.M.; Groffen, J.; Bootsma, D.; Grosveld, G. Unique fusion of BCR and C-ABL genes in Philadelphia chromosome positive acute lymphoblastic leukemia. Cell 1987, 51, 33-40.

78. Ben-Neriah, Y.; Daley, G.Q.; Mes-Masson, A.M.; Witte, O.N.; Baltimore, D. The chronic myelogenous leukemia-specific P210 protein is the product of the bcr/abl hyrbrid gene. Science 1986, 233, 212-214.

79. Sánchez-García, I.; Grütz, G. Tumorigenic activity of the BCR-ABL oncogenes is mediated by BCL2. Proc. Natl. Acad. Sci. USA 1995, 92, 5287-5291.

80. Wittrup, A.; Lieberman, J. Knocking down disease: A progress report on siRNA therapeutics. Nat. Rev. Genet. 2015, 16, 543-552.

81. Zhang, H.; Altman, S. Inhibition of the expression of the human RNase P protein subunits Rpp21, Rpp25, Rpp29 by external guide sequences (EGSs) and siRNA. J. Mol. Biol. 2004, 342, 1077-1083.

82. Gibbons, D.L.; Shashikant, C.; Hayday, A.C. A comparative analysis of RNA targeting strategies in the thymosin beta 4 gene. J. Mol. Biol. 2004, 342, 1069-1076.

(C) 2015 by the authors; licensee MDPI, Basel, Switzerland. This article is an open access article distributed under the terms and conditions of the Creative Commons Attribution license (http://creativecommons.org/licenses/by/4.0/). 